\title{
Network Structures of Social Functioning Domains in Schizophrenia and Bipolar Disorder: A Preliminary Study
}

\author{
Seunghyong Ryu', Hyeongrae Lee ${ }^{2}$, Dong-Kyun Lee ${ }^{2}$, Hee Jung Nam $^{3}$, Young-Chul Chung ${ }^{4}$, Sung-Wan Kim ${ }^{1}$ \\ ${ }^{1}$ Department of Psychiatry, Chonnam National University Medical School, Gwangju, ${ }^{2}$ Department of Mental Health Research, National Center \\ for Mental Health, ${ }^{3}$ Department of Psychiatry, Seoul Medical Center, Seoul, ${ }^{4}$ Department of Psychiatry, Chonbuk National University Medical \\ School, Jeonju, Korea
}

\begin{abstract}
Objective: This study used network analyses to examine network structures reflecting interactions between specific domains of social functioning in schizophrenia (SZ) and bipolar disorder (BD).

Methods: We used the World Health Organization Disability Assessment Schedule 2.0 (WHODAS 2.0) to assess six domains of social functioning ('cognition', 'mobility', 'self-care', 'getting along', 'life activities', and 'participation') in 143 patients with SZ, 81 patients with BD, and 106 healthy subjects. We constructed regularized partial correlation networks, estimated network centrality and edge strength, tested network stability, and compared SZ and BD network structures.

Results: Patients with SZ showed a significantly higher level of functional disability than patients with BD. In the networks we constructed, 'cognition' was the most central domain of social functioning in both SZ and BD. The 'cognition' domain was primarily associated with the 'getting along' domain in the SZ network and the 'life activities' domain in the BD network. We found no significant group-level differences in network structures for SZ vs. BD.

Conclusion: Our results suggest that cognition may play a pivotal role in social functioning in both SZ and BD. In addition, domains of social functioning in SZ and BD have similar network structures despite the higher level of disability in SZ compared to BD.
\end{abstract}

KEY WORDS: Network; Social abilities; Schizophrenia; Bipolar disorder; Cognition.

\section{INTRODUCTION}

Impairment of social functioning is a common feature of severe mental illnesses and is the leading contributor to disease burden in patients and their families [1,2]. Schizophrenia (SZ) is characterized by functional deficits, including lack of self-care, social isolation, and an inability to work $[3,4]$. These deficits are typically present from the early stages of the illness, and become more pronounced with repeated relapses [5]. Patients with bipolar disorder (BD) have historically been thought to retain a standard level of function between episodes and to have

Received: February 27, 2020 / Revised: March 30, 2020

Accepted: March 31, 2020

Address for correspondence: Sung-Wan Kim

Department of Psychiatry, Chonnam National University Medical

School, 42 Jebong-ro, Dong-gu, Gwangju 61469, Korea

E-mail: swkim@chonnam.ac.kr

ORCID: https://orcid.org/0000-0002-6739-2163 better outcomes than SZ patients. However, recent studies have suggested a significant degree of social dysfunction in patients with $\mathrm{BD}$, even during periods of clinical remission $[6,7]$.

Social functioning is a complex concept, consisting of the capacity to work, study, live independently, and engage in recreational activities and interpersonal relationships [8]. Thus, impairment of social functioning could be defined as the inability of an individual to fill their social roles (e.g., worker, homemaker, student, family member, or friend). A person with mental illnesses experiences functional difficulties at the physical, personal, or societal level in one or more domains [9]. Individual domains of social functioning may interact with each other to collectively determine the patient's overall level of function. Impairment in one domain may lead to functional deterioration in other domains. By contrast, relatively healthy function in one domain can compensate for challenges in

(c) This is an Open-Access article distributed under the terms of the Creative Commons Attribution Non-Commercial License (http://creativecommons.org/licenses/by-nc/4.0) which permits unrestricted non-commercial use, distribution, and reproduction in any medium, provided the original work is properly cited. 
other domains. Therefore, it is necessary to obtain a comprehensive picture of a patient's functional disabilities to develop targeted clinical interventions to improve their social functioning.

Little is known about the relationships between individual domains of social functioning in patients with SZ and $\mathrm{BD}$, although several factors have been associated with poor functional outcomes (e.g., neurocognitive impairment, persistent negative symptoms, subsyndromal mood symptoms, and side effects of pharmacological treatment). Therefore, this study used network analysis to explore patterns of connectivity between individual domains of social functioning in patients with $\mathrm{SZ}$ and $\mathrm{BD}$. We assessed individual domains of social functioning using the World Health Organization Disability Assessment Schedule 2.0 (WHODAS 2.0) [10] in patients with SZ and BD. Network analysis is a powerful methodological approach that can be used to estimate complex patterns of interconnected variables and obtain visual depictions of system-level relationships [11]. This approach has the potential to show how individual domains of social functioning influence each other and which domains play a pivotal role in the functioning of patients with SZ and BD. We also compared estimated network structures and overall level of social functioning in $\mathrm{SZ}$ and $\mathrm{BD}$ by identifying qualitative and quantitative differences in patients' social functioning.

\section{METHODS}

\section{Subjects}

We recruited subjects for the present study via offline advertisements at the National Center for Mental Health between July 2017 and August 2019. Inclusion criteria for patients were as follows: 1 ) aged $20-50$ years; 2 ) meeting all diagnostic criteria for $\mathrm{SZ}$ or $\mathrm{BD}$ type I or II, as defined in the Diagnostic and Statistical Manual of Mental Disorders IV (DSM-IV); 3) having a duration of illness greater than one year; and 4) appearing clinically stable, defined as no exacerbation of psychotic symptoms and no change in general clinical state and medication in the three months preceding the assessment. Patients with other psychiatric disorders, neurological diseases, or physical conditions that would affect social functioning were excluded. To determine the level of functional disability in patients with $\mathrm{SZ}$ and BD compared to healthy people, we also recruited healthy subjects in the same age range with no history of psychiatric disorders. As in our patient sample, we excluded people with other psychiatric disorders, neurological diseases, or physical conditions that would affect social functioning. In total, 330 subjects (143 patients with SZ, 81 patients with BD, and 106 healthy people) were found to be eligible for the present study.

This study was initiated after receiving the approval of the Institutional Review Board of the National Center for Mental Health (IRB approval no. 116271-2017-26). Written informed consent was obtained from all subjects.

\section{Measurements}

\section{Mini-International Neuropsychiatric Interview}

All subjects were interviewed by psychologists using the Korean version of the Mini-International Neuropsychiatric Interview (MINI). The MINI is a structured diagnostic interviewing tool based on the DSM-IV and the International Classification of Diseases 10th Revision [12].

\section{WHO Disability Assessment Schedule 2.0}

We assessed the patients' social functioning using a Korean translation of the 36-item interviewer-administered version of WHODAS 2.0. The WHODAS 2.0 is a multidimensional instrument designed to assess functioning in life and community activities over six sub-domains: 'cognition', 'mobility', 'self-care', 'getting along', 'life activities', and 'participation' $[10,13]$. The WHODAS 2.0 has high internal consistency, high test-retest reliability, and good concurrent validity compared to other recognized disability measurement instruments. Respondents were asked to respond to each of 36 items based on their recollection of the previous 30 days, using a five-point Likert scale ranging from one (no disability) to five (extreme disability or unable to do). The WHODAS 2.0 provides simple and complex options for computing summary scores. In this study, we employed the complex option by converting the sum of all six domain scores into a score ranging from 0 to 100 , with a higher score indicating greater disability.

\section{8-item Brief Psychiatric Rating Scale}

An experienced psychiatrist evaluated the overall severity of patients' psychopathology using the 18-item Brief Psychiatric Rating Scale (BPRS-18) [14]. The BPRS-18 
consists of 18 items that are scored on a seven-point Likert scale, with higher scores indicating more severe psychopathological symptoms.

\section{Network Analyses}

\section{Network construction}

We assumed a network consisting of the six sub-domains of the WHODAS 2.0, with individual domains of social functioning represented as nodes (D1 to D6) and associations between them represented as edges. For the edges, we calculated partial correlations between two nodes after controlling for all other relations in the network. We estimated the network using the Gaussian graphical model (GGM) [15], in which undirected edges indicate conditional dependence relations between nodes. To limit the number of spurious correlations, we applied the graphical least absolute shrinkage and selection operator (LASSO) to the GGM [16]. This statistical regularization technique shrinks edges and sets small edges to zero, leading to a sparse network structure that balances parsimony with explanatory power. We carried out this process using the R package 'qgraph', which automatically implements graphical LASSO regularization in combination with Extended Bayesian Information Criterion (EBIC) model selection [17]. We set the value of the hyperparameter $\gamma$, which denotes the strength of the EBIC's preference for sparser models, to 0.5 [18]. This reflects a balance between a spurious network $(\gamma=0)$ and a more parsimonious network $(\gamma=1)$. The nodes were positioned using the Fruchterman-Reingold algorithm [19]. This algorithm calculates the optimal layout for placing nodes with strong connections close to each other while placing those with weaker and fewer connections farther apart. The thickness of the edges represents the strength of the association, with thicker lines representing stronger associations.

In addition, we used the R package 'igraph' to calculate macroscopic properties of the SZ and BD networks and obtain information about the overall connectedness of the network. The average shortest path length is an important measure of a network's overall connectedness [20]. The diameter of a network corresponds to the longest shortest path length [21], and the density of a network is the actual number of edges as a proportion of the total possible number of edges [20]. Finally, the average clustering coefficient, which indicates the extent to which nodes tend to cluster together, is an average of the local clustering coefficients and the ratio between triangles connected to the vertex and triples centered on the vertex [22]. A network with a higher density, a higher average clustering coefficient, and a lower average shortest path length can be regarded as tightly interconnected [23].

\section{Centrality estimation}

Centrality indices provide insight into the relative importance of a node in the context of the other nodes in the network $[24,25]$. To identify which domains of social functioning measured by the WHODAS 2.0 were most central to the network, we used the R package 'qgraph' to assess three centrality indices for each node within the network: strength, closeness, and betweenness. Node strength indicates the strength of a node's direct connections to other nodes, and is calculated as the sum of the weighted number and strength of all connections of a specific node relative to all other nodes [26]. Node closeness represents the average distance from a node to all other nodes in the network, with high closeness indicating a short average distance between a given node and the remaining nodes in the network [25]. Node betweenness measures the number of times that a node lies on the shortest path between two other nodes, and provides information on how important a node is in the average pathway between another pair of nodes [27]. In addition, we tested for significant differences between nodes in centrality indices using the bootstrapped difference tests.

\section{Network stability and accuracy}

We examined the stability and accuracy of the network using the R package 'bootnet'. The stability of the centrality indices was examined using the case-dropping subset bootstrap (2,500 iterations), which determines how many samples can be removed from the network before the results become unstable [28]. This measure is quantified by the correlation stability (CS) coefficient. The CS coefficient represents the maximum proportion of cases that can be dropped while retaining a correlation of 0.7 between original centrality indices and centrality based on subset [28]. It is suggested that the CS coefficient be at least 0.25 , and preferably above 0.5 [29]. To assess the accuracy of the edge weights, we drew 2,500 bootstrapped confidence intervals (Cls) of the edge weights. Smaller Cls indicate more accurate estimates of the edge 
weights. $\mathrm{A} \mathrm{Cl}$ that crosses zero indicates that the edge weight on which the $\mathrm{Cl}$ is based is not significantly different from other edge weights. We confirmed significant differences between edge weights using the bootstrapped difference test.

\section{Network comparison}

We compared SZ and BD networks of social functioning using the R package 'NetworkComparisonTest', a permutation-based hypothesis test in which the difference between networks of two groups is calculated repeatedly for randomly regrouped individuals [30]. We performed 2,500 iterations to assess the difference between the two networks through comparisons of network structure, global strength, and edge invariance.

\section{RESULTS}

\section{Sample Characteristics}

Table 1 summarizes the demographic and clinical characteristics of the study sample. There were no significant difference in sex for the SZ, BD, and healthy subjects groups. However, healthy subjects were significantly older and better educated than patients with SZ and BD. Duration of illness and treatment doses of antipsychotic drugs were significantly higher in the SZ group than in the BD group. Patients with SZ showed significantly higher total scores on the BPRS-18 than patients with BD. Total scores on the WHODAS 2.0 were significantly higher for patients with SZ vs. patients with BD and healthy subjects, and significantly higher for patients with BD vs. healthy subjects. In particular, even after controlling for duration of illness and doses of antipsychotic drugs as covariates, the difference in total scores on the WHODAS 2.0 between the $S Z$ and $B D$ groups was still significant $(B=-6.83, \beta=$ $-0.34, p<0.001)$. In addition, the BD group included 67 patients with BD type I (82.72\%) and 14 patients with BD type II (17.28\%). Fifty patients with BD (61.73\%) had a history of psychotic features.

\section{Network of Social Functioning Domains}

The SZ and BD networks of the six domains of social functioning are presented in Figure 1. All nodes within the networks were positively connected. The SZ network had an average shortest path length of 1.33, a diameter of 0.52 , a density of 0.67 , and an average clustering coefficient of 0.81. Similarly, the BD network had an average shortest path length of 1.53 , a diameter of 0.71 , a density of 0.53 , and an average clustering coefficient of 0.70 .

Figure 2 depicts the standardized centrality indices of the networks. The centrality indices indicated that node D1 ('cognition') was the most central domain of social functioning in both the SZ network and the BD network. All nodes other than node D1 showed relatively lower centrality indices in the SZ network. In the BD network, node D5 ('life activities') showed node strength and closeness comparable to node D1. In the SZ network, the bootstrap significance test revealed that node D1 differed sig-

Table 1. Demographic and clinical characteristics

\begin{tabular}{|c|c|c|c|c|}
\hline Variables & $\begin{array}{l}\text { Healthy control } \\
\qquad(n=106)\end{array}$ & $\begin{array}{l}\text { Schizophrenia } \\
\qquad(\mathrm{n}=143)\end{array}$ & $\begin{array}{l}\text { Bipolar disorder } \\
\qquad(\mathrm{n}=81)\end{array}$ & Statistics $^{\mathrm{a}}$ \\
\hline Age (yr) & $31.38 \pm 8.92$ & $36.69 \pm 8.24$ & $34.57 \pm 8.93$ & $\mathrm{~F}=11.55, p<0.001$ \\
\hline Sex (male/female) & $45 / 61$ & $76 / 67$ & $39 / 42$ & $\chi^{2}=2.79, p=0.248$ \\
\hline Education (yr) & $14.54 \pm 2.50$ & $12.55 \pm 2.56$ & $13.96 \pm 1.89$ & $\mathrm{~F}=22.68, p<0.001$ \\
\hline Duration of illness (yr) & - & $15.56 \pm 8.04$ & $13.19 \pm 8.02$ & $t=2.13, p=0.034$ \\
\hline \multicolumn{5}{|l|}{ Medication } \\
\hline Antipsychotics & - & 143 & 71 & $\chi^{2}=18.48, p<0.001$ \\
\hline Chlorpromazine equivalent dose (mg) & - & $813.80 \pm 501.14$ & $344.49 \pm 316.64$ & $t=7.61, p<0.001$ \\
\hline Mood stabilizers & - & 44 & 62 & $\chi^{2}=43.46, p<0.001$ \\
\hline Valproate/lithium/lamotrigine & - & $31 / 16 / 2$ & $39 / 26 / 9$ & - \\
\hline Antidepressants & - & 31 & 16 & $\chi^{2}=0.12, p=0.734$ \\
\hline BPRS-18 total score & - & $38.13 \pm 9.19$ & $28.17 \pm 7.80$ & $t=8.21, p<0.001$ \\
\hline WHODAS 2.0 total score & $0.80 \pm 1.30$ & $19.94 \pm 9.55$ & $11.03 \pm 7.01$ & $\mathrm{~F}=214.12, p<0.001$ \\
\hline
\end{tabular}

Values are presented as mean \pm standard deviation or number only. BPRS-18, 18-item Brief Psychiatric Rating Scale; WHODAS 2.0, World Health Organization Disability Assessment Schedule 2.0; -, not available. ${ }^{a}$ ANOVA, chi-square test, or independent $t$ test. 
A Schizophrenia

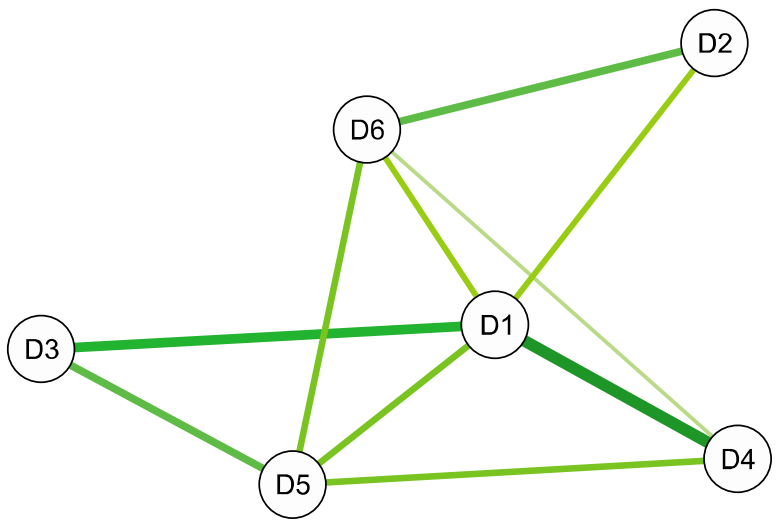

B Bipolar disorder

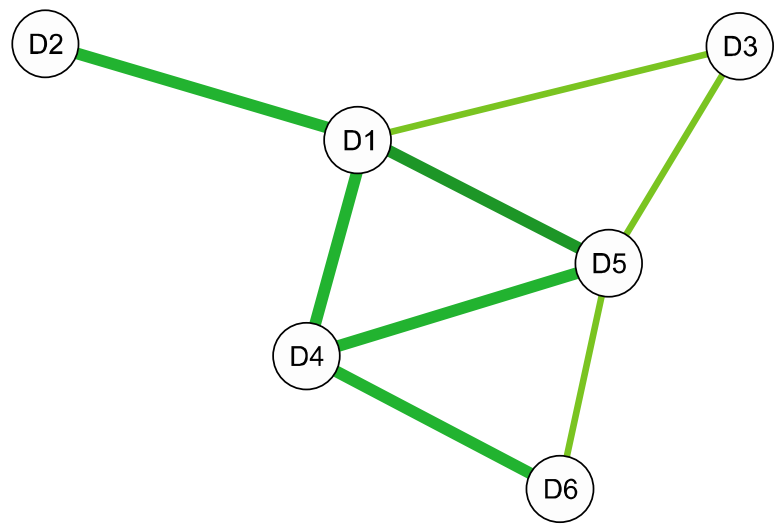

Fig. 1. Regularized partial correlation networks of the six domains of the WHODAS 2.0. Green edges denote positive correlations. The thickness of the edges represents the magnitude of the correlation.

D1, cognition; D2, mobility; D3, self-care; D4, getting along; D5, life activities; D6, participation; WHODAS 2.0, World Health Organization Disability Assessment Schedule 2.0.

A Schizophrenia

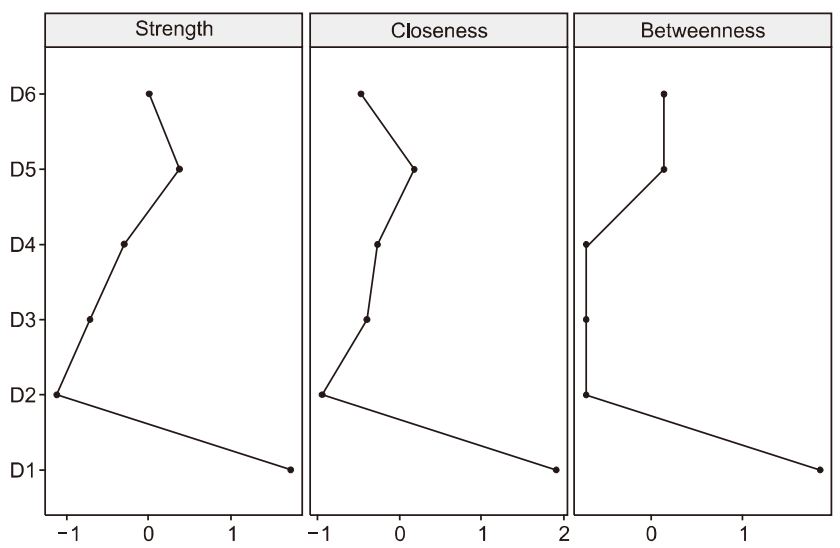

B Bipolar disorder

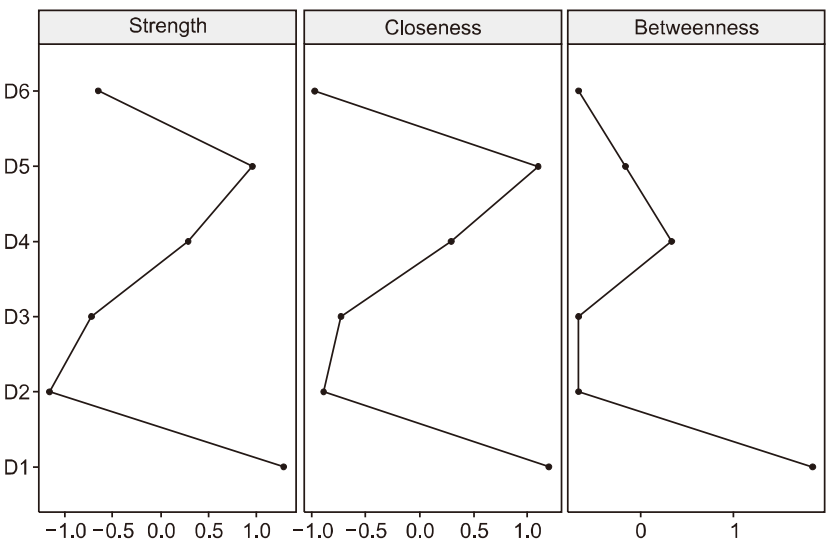

Fig. 2. Centrality indices of the six domains of the WHODAS 2.0. Centrality indices are shown as standardized z-scores.

D1, cognition; D2, mobility; D3, self-care; D4, getting along; D5, life activities; D6, participation; WHODAS 2.0, World Health Organization Disability Assessment Schedule 2.0.

nificantly from D2 ('mobility') in node strength and closeness and from D4 ('getting along') in node strength in the SZ network. There were no significant differences in centrality measures for the nodes in the BD network.

Figure 3 shows the stability of centrality indices. The results of the case-dropping subset bootstrap revealed low CS coefficients (below 0.25) for all centrality indices in both the SZ and BD networks, suggesting that the results should be interpreted with caution.

Figure 4 presents the accuracy of the edge weights with 95\% Cls. The strongest edge in the SZ network was found between nodes D1 and D4 ('cognition' and 'getting along', 0.37), followed by the edge between nodes D1 and D3 ('cognition' and 'self-care', 0.31). The Cls for these edges did not contain zero. Bootstrap significance tests confirmed that the edge between D1 and D4 had a significantly stronger edge weight than the edge between D4 and D6 ('getting along' and 'participation'). In the BD network, the strongest edge was between nodes D1 and D5 ('cognition' and 'life activities', 0.28), followed by the edge between D1 and D2 ('cognition' and 'mobility', 0.26). However, the Cls for these edges contained zero.

The comparison of the SZ and BD networks showed no significant difference in network structure (test statistic $=$ $0.25, p=0.925$ ) or global strength (test statistic $=0.56, p=$ 0.218 ). Since there was no significant difference in net- 

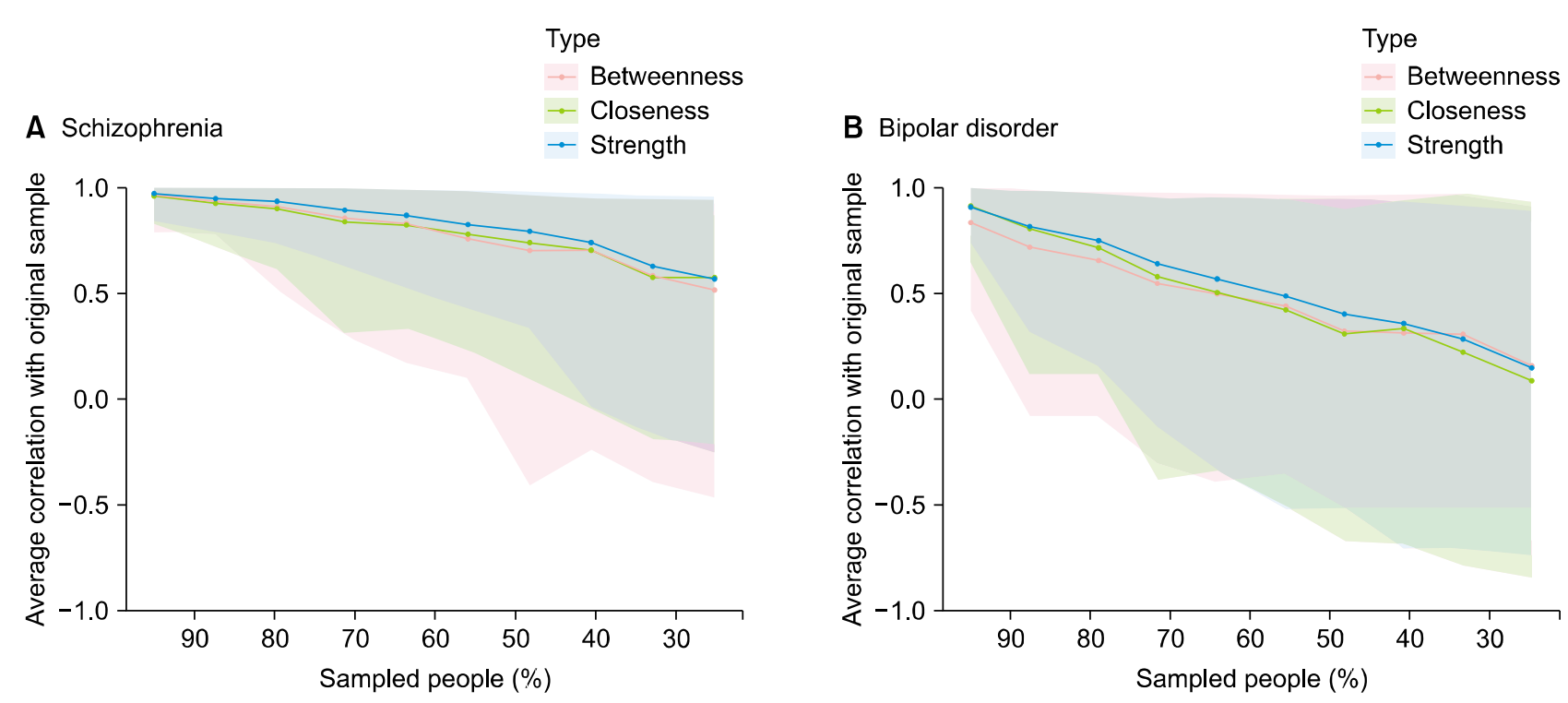

Fig. 3. Stability of centrality indices.
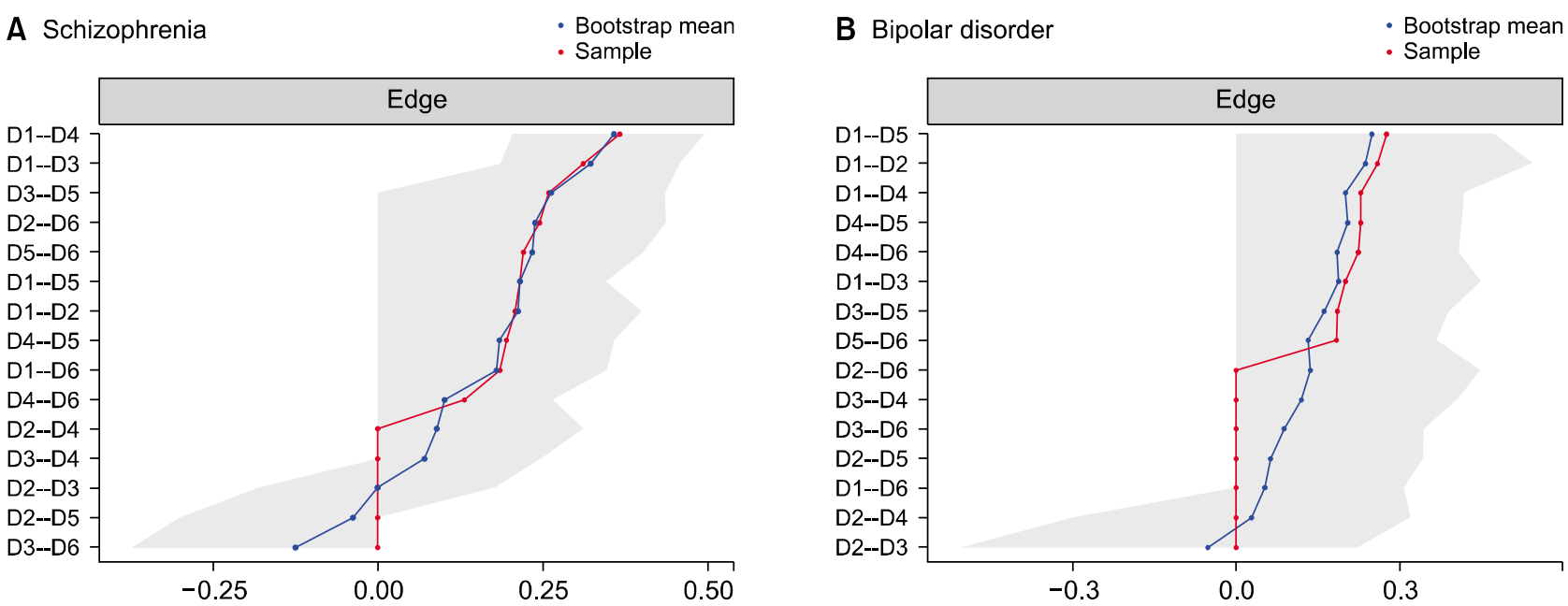

Fig. 4. Accuracy of edge weights with $95 \%$ confidence intervals.

D1, cognition; D2, mobility; D3, self-care; D4, getting along; D5, life activities; D6, participation.

work structure, we did not perform further testing of specific edge invariances.

\section{DISCUSSION}

The present study constructed network structures for SZ and $\mathrm{BD}$ based on the six domains of social functioning measured by the WHODAS 2.0. Networks were created using regularized partial correlation and estimates of the central indices of nodes and the strength of edges within the networks. The results showed that 'cognition' was the core domain of social functioning. In both SZ and BD, 'cognition' was the domain that was most often directly or indirectly connected with other domains. We found no significant differences in the network structures of functional domains in SZ and BD, although total scores on the WHODAS 2.0 showed that patients with SZ had more severe functional disability than patients with BD. To our knowledge, this is the first study to describe relationships between specific domains of social functioning in SZ and BD using the network approach.

In both the SZ and BD networks, we found that 'cognition' was the sub-domain of the WHODAS 2.0 with the highest centrality indices, including node strength, 
closeness, and betweenness. In terms of edge strength, this domain had a significant association with the 'getting along' domain in the SZ network. The 'cognition' domain of the WHODAS 2.0 does not directly represent neurocognitive functions (e.g., attention, memory), but consists of items asking about understanding and communicating in everyday life $[10,13]$. These items are closely linked to neurocognitive functions. Cognitive impairment is a substantial clinical problem among patients with BD and patients with SZ, although it is more severe among patients with SZ [31]. Cognitive deficits may cause difficulties in acquiring and deploying social skills and life skills, and consequently impede functional recovery in patients with $\mathrm{SZ}$ and BD. Our findings are compatible with previous research showing that neurocognitive functions are associated with functional outcomes in both SZ and BD $[32,33]$. The present study suggests that cognitive impairment should be considered a potential target in the treatment of functional disabilities in patients with SZ and BD, and that cognitive rehabilitation could be an effective intervention in these patients.

In this study, comparisons of total scores on the WHODAS 2.0 showed that patients with SZ and BD all had significant impairment in social functioning compared to healthy subjects, and that patients with SZ experienced more severe disability than patients with BD. However, network analysis revealed that networks based on functional domains were similar for SZ and BD. As stated above, 'cognition' was the salient domain of social functioning in both the SZ and BD networks. Moreover, a direct comparison of network structures did not reveal any significant differences between the two disorders. In other words, the relationships between domains of social functioning were similar for SZ and BD. These findings suggest a common process leading to functional impairment in SZ and BD, although patients with SZ have more pronounced impairment of social functioning than patients with BD.

In this study, we sought to understand social functioning in patients with SZ and BD as a system of interconnected domains using the network approach. From a network perspective, human behaviors and outcomes can be conceptualized as emergent phenomena from a system of reciprocal interactions of relevant variables [29]. Network analysis is a promising method for examining the complex patterns of these relationships and generating graphical representations that conventional statistical methods cannot provide. Network analysis is applied increasingly often in the fields of psychopathology, personality, and health psychology [34-37]. This study demonstrates that network analysis may offer novel insights into social functioning in patients with SZ and BD.

The present study has some methodological limitations. First, the study subjects were heterogeneous in their demographic and clinical characteristics, which might have influenced their functional status. Patients with SZ and BD showed a wide range in the duration of illness and type of medication. In addition, we included patients with all types of BD, regardless of whether they were type I, type II, or had a history of psychosis. Second, the network analysis in this study did not account for functional deterioration over time in patients with $\mathrm{SZ}$ and $\mathrm{BD}$; we assessed social functioning only at the cross-sectional level. Third, we could not generate a network of social functioning for healthy subjects because their domain scores on the WHODAS 2.0 were too low to be correlated with each other. Fourth, bootstrapping tests for stability and accuracy indicated that the network structures modeled in this study should be interpreted with caution. The CS coefficients were lower than recommended and numerous Cls for edge weight contained zero, suggesting that the stability of the central indices and the accuracy of edge strengths might be unreliable [29]. Therefore, the results of the present study should be considered preliminary findings. In psychology networks, centrality indices tend to reach the threshold for reliable estimation in large samples [38]. Therefore, further studies with larger sample sizes are needed.

Despite these limitations, the present study shows that cognition plays a key role in social functioning in both SZ and BD. This suggests that cognitive rehabilitation should be considered as a method for improving functional outcomes and reducing disability in patients with SZ and BD. In addition, we found that the social functioning domains in SZ and BD share similar network structures. We could hypothesize that common mechanisms contribute to functional impairment in the two disorders. Future studies of the network of social functioning and its relationship to psychopathology and clinical outcomes are warranted.

\section{- Acknowledgments}

This study was supported by a grant from the Basic 
Science Research Program through the National Research Foundation of Korea grant funded by the Korea government (MSIT) (NRF-2017R1A2B4010830).

\section{- Conflicts of Interest}

No potential conflict of interest relevant to this article was reported.

\section{Author Contributions}

Conceptualization: Seunghyong Ryu, Young-Chul Chung, and Sung-Wan Kim. Data acquisition: Hyeongrae Lee, Dong-Kyun Lee. Formal analysis: Seunghyong Ryu, Hyeongrae Lee, and Dong-Kyun Lee. Funding: Sung-Wan Kim. Supervision: Young-Chul Chung. Writing-original draft: Seunghyong Ryu, Hee Jung Nam, Sung-Wan Kim. Writing - review \& editing: Seunghyong Ryu, Sung-Wan Kim.

\section{ORCID}

Seunghyong Ryu https://orcid.org/0000-0001-6127-760X Hyeongrae Lee https://orcid.org/0000-0002-5146-680X Dong-Kyun Lee https://orcid.org/0000-0001-7191-000X Hee Jung Nam https://orcid.org/0000-0001-8375-7516 Young-Chul Chung https://orcid.org/0000-0001-9491-1822 Sung-Wan Kim https://orcid.org/0000-0002-6739-2163

\section{REFERENCES}

1. Simons CJP, Bartels-Velthuis AA, Pijnenborg GHM; Genetic Risk and Outcome of Psychosis (GROUP) Investigators. Correction: cognitive performance and long-term social functioning in psychotic disorder: a three-year follow-up study. PLoS One 2018;13:e0208347.

2. Lee RS, Hermens DF, Naismith SL, Lagopoulos J, Jones A, Scott J, et al. Neuropsychological and functional outcomes in recent-onset major depression, bipolar disorder and schizophrenia-spectrum disorders: a longitudinal cohort study. Transl Psychiatry 2015;5:e555.

3. Harvey PD, Reichenberg A, Bowie CR, Patterson TL, Heaton RK. The course of neuropsychological performance and functional capacity in older patients with schizophrenia: influences of previous history of long-term institutional stay. Biol Psychiatry 2010;67:933-939.

4. Tripathi A, Kar SK, Shukla R. Cognitive deficits in Schizophrenia: understanding the biological correlates and remediation strategies. Clin Psychopharmacol Neurosci 2018;16:7-17.

5. Lieberman JA. Is schizophrenia a neurodegenerative disorder? A clinical and neurobiological perspective. Biol Psychiatry 1999:46:729-739.
6. Bowie CR, Best MW, Depp C, Mausbach BT, Patterson TL, Pulver $\mathrm{AE}$, et al. Cognitive and functional deficits in bipolar disorder and schizophrenia as a function of the presence and history of psychosis. Bipolar Disord 2018;20:604-613.

7. Tohen M, Hennen J, Zarate CM Jr, Baldessarini RJ, Strakowski $\mathrm{SM}$, Stoll AL, et al. Two-year syndromal and functional recovery in 219 cases of first-episode major affective disorder with psychotic features. Am J Psychiatry 2000;157:220-228.

8. Zarate CA Jr, Tohen M, Land M, Cavanagh S. Functional impairment and cognition in bipolar disorder. Psychiatr $Q$ 2000;71:309-329.

9. Leonardi M, Bickenbach J, Ustun TB, Kostanjsek N, Chatterji S; MHADIE Consortium. The definition of disability: what is in a name? Lancet 2006;368:1219-1221.

10. Ustün TB, Chatterji S, Kostanjsek N, Rehm J, Kennedy C, Epping-Jordan J, et al. Developing the World Health Organization disability assessment schedule 2.0. Bull World Health Organ 2010;88:815-823.

11. Borgatti SP, Mehra A, Brass DJ, Labianca G. Network analysis in the social sciences. Science 2009;323:892-895.

12. Sheehan DV, Lecrubier $Y$, Sheehan $\mathrm{KH}$, Amorim $\mathrm{P}$, Janavs J, Weiller E, et al. The Mini-International Neuropsychiatric Interview (M.I.N.I.): the development and validation of a structured diagnostic psychiatric interview for DSM-IV and ICD-10. J Clin Psychiatry 1998;59 Suppl 20:22-33;quiz 34-57.

13. Gold LH. DSM-5 and the assessment of functioning: the World Health Organization Disability Assessment Schedule 2.0 (WHODAS 2.0). J Am Acad Psychiatry Law 2014;42. 173-181.

14. Overall JE, Gorham DR. The Brief Psychiatric Rating Scale (BPRS): recent developments in ascertainment and scaling. Psychopharmacol Bull 1988;24:97-9.

15. Lauritzen SL. Graphical models. Oxford:Clarendon Press; 1996.

16. Friedman J, Hastie T, Tibshirani R. Sparse inverse covariance estimation with the graphical lasso. Biostatistics 2008;9:432-441.

17. Chen JH, Chen ZH. Extended Bayesian information criteria for model selection with large model spaces. Biometrika 2008; 95:759-771.

18. Epskamp S, Fried El. A tutorial on regularized partial correlation networks. Psychological Methods 2018;23:617-634.

19. Fruchterman TMJ, Reingold EM. Graph drawing by force-directed placement. Software 1991;21:1129-1164.

20. Rubinov M, Sporns O. Complex network measures of brain connectivity: uses and interpretations. Neuroimage 2010;52: 1059-1069.

21. Csardi G, Nepusz T. The igraph software package for complex network research. Interjournal Complex Syst 2006;1695:1-9.

22. Watts DJ, Strogatz SH. Collective dynamics of 'small-world' networks. Nature 1998;393:440-442.

23. Esfahlani FZ, Sayama H, Visser KF, Strauss GP. Sensitivity of the Positive and Negative Syndrome Scale (PANSS) in detecting treatment effects via network analysis. Innov Clin NeurosCi 2017; 14:59-67. 
24. Freeman LC. Centrality in social networks conceptual clarification. Soc Netw 1978;1:215-239.

25. Borgatti SP. Centrality and network flow. Soc Netw 2005;27: 55-71.

26. Opsahl T, Agneessens F, Skvoretz J. Node centrality in weighted networks: generalizing degree and shortest paths. Soc Netw 2010;32:245-251.

27. Saramäki J, Kivelä M, Onnela JP, Kaski K, Kertész J. Generalizations of the clustering coefficient to weighted complex networks. Phys Rev E Stat Nonlin Soft Matter Phys 2007;75(2 Pt 2):027105.

28. Epskamp S, Borsboom D, Fried El. Estimating psychological networks and their accuracy: a tutorial paper. Behav Res Methods 2018;50:195-212.

29. Hevey D. Network analysis: a brief overview and tutorial. Health Psychol Behav Med 2018;6:301-328.

30. van Borkulo C, Boschloo L, Kossakowski J, Tio P, Schoevers R, Borsboom D, et al. Comparing network structures on three aspects: a permutation test. ResearchGate [Preprint]. 2017 [cited 2020 Feb 1]. Available from: https://doi.org/10.13140/ RG.2.2.29455.38569.

31. Harvey PD, Wingo AP, Burdick KE, Baldessarini RJ. Cognition and disability in bipolar disorder: lessons from schizophrenia research. Bipolar Disord 2010;12:364-375.

32. Martinez-Aran A, Vieta E, Torrent C, Sanchez-Moreno J, Goikolea JM, Salamero M, et al. Functional outcome in bipo- lar disorder: the role of clinical and cognitive factors. Bipolar Disord 2007;9:103-113.

33. Simons CJ, Bartels-Velthuis AA, Pijnenborg GH; Genetic Risk and Outcome of Psychosis (GROUP) Investigators. Cognitive performance and long-term social functioning in psychotic disorder: a three-year follow-up study. PLoS One 2016;11: e0151299.

34. Borsboom D. A network theory of mental disorders. World Psychiatry 2017;16:5-13.

35. Kossakowski JJ, Epskamp S, Kieffer JM, van Borkulo CD, Rhemtulla M, Borsboom D. The application of a network approach to Health-Related Quality of Life (HRQoL): introducing a new method for assessing HRQoL in healthy adults and cancer patients. Qual Life Res 2016;25:781-792.

36. Richetin J, Preti E, Costantini G, De Panfilis C. The centrality of affective instability and identity in Borderline Personality Disorder: evidence from network analysis. PLoS One 2017; 12:e0186695.

37. Murphy J, McBride O, Fried E, Shevlin M. Distress, impairment and the extended psychosis phenotype: a network analysis of psychotic experiences in an US general population sample. Schizophr Bull 2018;44:768-777.

38. Santos HP Jr, Kossakowski JJ, Schwartz TA, Beeber L, Fried El. Longitudinal network structure of depression symptoms and self-efficacy in low-income mothers. PLoS One 2018;13: e0191675. 